\title{
Efeitos da suplementação e da fortificação de alimentos sobre a biodisponibilidade de minerais
}

\section{Effects of supplementation and food fortification on mineral bioavailability}

Adriana Soares $\mathrm{LOBO}^{1}$

Vera Lúcia Cardoso TRAMONTE²

RE S U M O

Estratégias de prevenção e combate a algumas deficiências nutricionais, como a anemia e a osteoporose, incluem a fortificação de alimentos e o uso de suplementação com minerais em populações de risco. Entretanto, interações com outros minerais podem ocorrer e comprometer o estado de saúde do indivíduo. Este artigo teve por objetivo rever algumas das interações que podem ocorrer entre minerais quando da suplementação ou fortificação de alimentos. A suplementação de cálcio parece estar relacionada a uma diminuição da absorção do zinco, fósforo e ferro. Por sua vez, o excesso de ferro pode comprometer a absorção e utilização do zinco, especialmente quando em formulações antianêmicas. Apesar dos resultados de alguns estudos serem controversos, a suplementação de minerais ou a fortificação de alimentos devem ser cuidadosas a fim de não ocasionar outras deficiências nutricionais.

Termos de indexação: biodisponibilidade biológica, minerais, suplementação, alimentos fortificados.

\section{A B S T R A C T}

Strategies for the prevention and control of some nutritional deficiencies, such as anemia and osteoporosis, include food fortification and the use of mineral supplements for at-risk populations. Nevertheless, interactions among minerals may occur and jeopardize the health status of subjects. The objective of this study was to review some possible interactions among minerals when supplementation and food fortification are used. Calcium supplements seem to be related to reduce zinc, phosphorus and iron absorption. In addition, iron excess can decrease zinc absorption and utilization, especially in antianemic formulas. Although several studies have shown controversial outcomes, mineral supplementation and food fortification must be carefully monitored, in order to avoid promoting other nutritional deficiencies.

Index terms: biological availability, minerals, supplementary, food, fortified.

\footnotetext{
1 Programa de Pós-Graduação em Nutrição, Universidade Federal de Santa Catarina. Campus Universitário, Caixa Postal 476, 88040-900, Santa Catariana, SC, Brasil. Correspondência para/Correspondence to: A.S. LOBO. E-mail: adrilobo@bol.com.br

2 Departamento de N utrição, Centro de Ciências da Saúde, Universidade Federal de Santa Catarina, Brasil. E-mail: veratramonte@ig.com.br
} 


\section{N T R O D U Ç Ã O}

Os minerais são elementos inorgânicos amplamente distribuídos na natureza e que, no organismo, desempenham uma variedade expressiva de funções metabólicas que incluem ativação, regulação, transmissão e controle ${ }^{1}$.

Uma melhor quantificação destes elementos em alimentos e fluidos biológicos, assim como o conhecimento dos mecanismos através dos quais exercem suas funções no organismo, só foram possíveis através do desenvolvimento de técnicas mais sensíveis e precisas que ocorreram a partir da década de setenta ${ }^{2}$.

Recentemente, com os estudos sobre biodisponibilidade, permitiu-se saber que 0 metabolismo dos minerais não pode ser considerado de maneira isolada. Fatores fisiológicos e nutricionais podem interferir na absorção, no transporte e no armazenamento, com subseqüente aumento da suscetibilidade à deficiência ou toxidade ${ }^{3}$.

Um dos fatores que interferem na biodisponibilidade dos minerais diz respeito às interações que ocorrem entre os mesmos ${ }^{2,3}$. De acordo com Couzi et al. ${ }^{4}$, as interações entre minerais podem ocorrer de forma direta ou indireta. A s interações diretas são geralmente fenômenos competitivos que ocorrem durante a absorção intestinal ou utilização tecidual, enquanto que as indiretas ocorrem quando um mineral está envolvido no metabolismo do outro, de modo que a deficiência de um acarreta num prejuízo de função do outro.

Apesar do conhecimento ainda ser limitado, algumas interações parecem estar bem estabelecidas e, sob algumas circunstâncias, podem ter implicações profundas na saúde humana 4 .

Como estratégias de combate a algumas deficiências nutricionais inclui-se a fortificação de alimentos com minerais e mesmo o uso de suplementação, em populações de risco. As possíveis interações entre estes micronutrientes devem ser consideradas a fim de não compro- meter o estado de saúde com relação a um outro mineral. Desta forma, pretende-se com este artigo rever algumas das interações que podem ocorrer entre minerais quando da suplementação ou fortificação de alimentos.

\section{Suplementação e fortificação de alimentos com cálcio}

Devido a relação entre deficiência de cálcio e osteoporose, a suplementação deste macromineral tem sido utilizada em mulheres adultas com vistas a minimizar perdas ósseas associadas à idade e ao desenvolvimento da osteoporose ${ }^{5}$. Da mesma forma, produtos alimentícios têm sido fortificados com cálcio, especialmente leite e produtos à base de leite ${ }^{6}$.

Entretanto, um potencial efeito adverso do cálcio quando oferecido com a refeição é a diminuição na absorção de minerais traços ${ }^{5}$. Ingestões elevadas de cálcio podem conduzir a uma diminuição na absorção de ferro ${ }^{5,7-9}$, fósforo ${ }^{10-12}$ e zinco ${ }^{6,12}$.

\section{Cálcio versus ferro}

Os efeitos da suplementação de cálcio sobre a absorção do ferro têm sido documentados em alguns estudos. Dawnson-Hughes et al. ${ }^{5}$ verificaram os efeitos da suplementação de $500 \mathrm{mg}$ de cálcio elementar (carbonato de cálcio e hidroxiapatita) sobre a absorção de $3,6 \mathrm{mg}$ de ferro não-heme em mulheres pós-menopausa. Os mesmos observaram uma redução de $50 \%$ a $60 \%$ na absorção do ferro de uma refeição (café da manhã) marcada extrinsecamente com ${ }^{59} \mathrm{Fe}$, contendo também $227 \mathrm{mg}$ de cálcio.

Da mesma forma, Cook et al. ${ }^{7}$ observaram uma redução na absorção do ferro não-heme de uma refeição composta por hambúrguer quando da suplementação de $600 \mathrm{mg}$ de cálcio na forma de citrato de cálcio ou fosfato de cálcio. Na forma de carbonato de cálcio não houve redução na absorção. 
A suplementação com diferentes doses de cálcio foi estudada por Hallberg et al. ${ }^{9}$ em adultos. Para tal, os autores utilizaram uma refeição teste (espécie de pão à base de farinha de trigo com manteiga) marcada com ${ }^{55} \mathrm{Fe} \mathrm{e}{ }^{59} \mathrm{Fe}$. As diferentes quantidades de cálcio na forma de cloridrato de cálcio $(40,75,165,300$ e $600 \mathrm{mg})$ foram adicionadas em duas diferentes séries: no preparo da massa da refeição, antes ou após do seu cozimento. Segundo Hallberg et al. ${ }^{9}$, acima de $300 \mathrm{mg}$, a inibição era diretamente relacionada a dose. Apesar da adição do cálcio antes do cozimento ter reduzido a fermentação do fitato, o que poderia ter interferido na absorção do ferro, sua absorção também foi significativamente diminuída quando o cálcio foi adicionado na refeição pronta, que continha pequena quantidade de fitato.

No mesmo estudo, os autores observaram que a absorção do ferro heme de uma refeição com hambúrguer foi marcadamente diminuída com $165 \mathrm{mg}$ de cálcio, sugerindo que o efeito do cálcio estaria relacionado a uma transferência de ferro na mucosa. Estes dados atentam para a importância de não se oferecer os suplementos de cálcio juntamente com as refeições que contenham ferro.

Neste sentido, Gleerup et al. ${ }^{8}$ procuraram verificar a possibilidade de diminuir a inibição do ferro não-heme pelo cálcio pela diminuição deste último no almoço e no jantar, haja vista que estas são as refeições em que normalmente são fornecidas as maiores quantidades de ferro durante o dia. Desta forma, $937 \mathrm{mg}$ de cálcio foram oferecidos diariamente a 21 mulheres em diferentes horários de ingestão. Ao final do experimento, os autores concluíram que a absorção poderia aumentar de 1,32mg para $1,76 \mathrm{mg}$ de ferro diário ( $34 \%$ ), se a ingestão de ferro se desse somente no desjejum e na ceia.

Em contrapartida, Reddy $\&$ Cook $^{13}$ não verificaram efeito significativo do cálcio sobre a absorção do ferro não-heme em uma dieta variada.
Apesar da maioria dos estudos ter evidenciado o potencial do cálcio em reduzir a absorção do ferro, Fairweather-Tait ${ }^{14}$ relata ainda que o fato mais importante na interação entre estes dois minerais diz respeito aos efeitos sobre os níveis de ferro corporais. Segundo alguns estudos citados pelo autor, suplementos de cálcio parecem não reduzir os estoques de ferro corporal, medidos pela concentração de ferritina plasmática.

\section{Cálcio versus zinco}

A interação entre cálcio e zinco vem merecendo a atenção de Dawnson-Hughes et al. ${ }^{5}$, no qual verificou o efeito suplementação de $500 \mathrm{mg}$ de cálcio elementar (carbonato de cálcio e hidroxiapatita) sobre o zinco (3,62 mg), não foi observada redução na absorção deste mineral.

Segundo Wood \& Zheng ${ }^{6}$, estudos sobre o assunto têm mostrado resultados controversos. Os mesmos autores avaliaram o efeito da ingestão de grandes quantidades de cálcio sobre a absorção do zinco em mulheres pós-menopausa. Estas receberam uma dieta padronizada contendo 17,6mg de zinco e 890mg de cálcio por dia e, após 12 dias, receberam mais $468 \mathrm{mg}$ de cálcio na forma de um alimento ou de um suplemento (fosfato de cálcio). o balanço de zinco foi significativamente reduzido durante o tratamento com altas doses de cálcio. Num segundo estudo, a ingestão de $600 \mathrm{mg}$ de cálcio junto com a refeição diminuiu a absorção de zinco em $50 \%$, concluindo que a dietas com altos teores de cálcio parecem aumentar as necessidades de zinco em adultos.

Mulheres durante a fase de lactação que receberam suplementos de cálcio (1000mg por dia) apresentaram concentrações plasmáticas de zinco similares às de mulheres que não receberam suplementação, sugerindo que o seu uso a longo prazo parece não ter efeito sobre o estado de zinco ${ }^{15}$.

Cozzolino $^{2}$ relata que a interação entre cálcio e zinco é mais pronunciada na presença de 
fitato. Na presença de cálcio, o complexo cálcio: fitato/zinco pode afetar adversamente o balanço de zinco em humanos, ocasionando problemas em dietas vegetarianas ou de populações de países em desenvolvimento, cuja ingestão de zinco é baixa e a de fitato é alta, quando numa relação molar maior que $200 \mathrm{mmol}$ por 1000 calorias.

Neste sentido, Walter et al. ${ }^{12}$ observaram que a interação cálcio-fitato-zinco pode prejudicar a biodisponibilidade de zinco em ratos em crescimento.

Apesar da relação cálcio-fitato-zinco ser postulada como um preditor da biodisponibilidade de zinco ${ }^{16}$, esta interação é bastante complexa e a relação pode ter valor preditivo limitado ${ }^{17}$. Lönnerdal et al. ${ }^{18}$ adicionaram cálcio em uma fórmula infantil à base de soja e observaram que a absorção de zinco aumentou significativamente quando comparada com a fórmula regular, sugerindo que a relação fitato-zinco seria mais importante para predizer a absorção do zinco do que a relação que compreende o cálcio.

\section{Cálcio versus fósforo}

O fósforo está intimamente associado ao cálcio na nutrição humana, sendo chamado de seu gêmeo metabólico. Desta forma, os fatores que favorecem ou dificultam a absorção do fósforo são praticamente os mesmos do cálcio. Para ajudar a manter o equilíbrio normal sérico cálcio-fósforo ${ }^{1}$, suas quantidades na dieta devem ser equilibradas em 1:1 $1^{1}$. Entretanto, suplementos de cálcio ou mesmo elevadas ingestões de cálcio podem comprometer este equilíbrio e alterar a absorção do fósforo.

Os efeitos do cálcio no metabolismo do fósforo foram estudados em adultos por Spencer et al. ${ }^{11}$, utilizando 200, 800 e $2000 \mathrm{mg} / \mathrm{dia}$ de cálcio e 200 e $800 \mathrm{mg} / \mathrm{dia}$ de fósforo. A adição de diferentes quantidades de cálcio levou a uma significante diminuição na excreção urinária de fósforo e a um aumento na excreção fecal de fósforo.
Al-M asri ${ }^{10}$ estudou a absorção e excreção endógena de fósforo em frangos alimentados com dieta contendo diferentes relações Ca:P (1:1; 1,5:1; $2: 1$ e 2,5:1) e observou que quanto maior esta proporção, menor eram a absorção e a excreção endógena e maior a retenção do fósforo.

Uma diminuição dose-dependente na absorção aparente de fósforo também foi evidenciada por W alter et al. ${ }^{12}$ em ratos suplementados com 2, 4, 6 e 8g/kg de ração de cálcio.

\section{Suplementação e fortificação de alimentos com ferro}

A anemia é a mais comum deficiência nutricional no mundo. Medidas preventivas e curativas baseiam-se na administração de sais de ferro como suplemento medicamentoso e/ou fortificação de alimentos com ferro ${ }^{19,20}$. Entretanto, esta prática pode exacerbar a deficiência de outros nutrientes, como o zinco ${ }^{21}$.

Conforme relatam Pedrosa $\&$ Cozzolino ${ }^{19}$, os agravantes neste aspecto são as formulações de medicamentos antianêmicos, onde constatamse níveis de ferro consideravelmente altos, excedendo as recomendações deste mineral para humanos.

A pesar dos efeitos adversos da suplementação de ferro sobre o zinco, Fairweather-Tait ${ }^{14}$ comenta que ainda existe uma incerteza acerca dos efeitos da fortificação de alimentos e suplementação de ferro na nutrição.

\section{Ferro versus Zinco}

A maioria dos estudos sobre a interação entre ferro e zinco tem sido feita utilizando-se diferentes níveis destes minerais administrados simultaneamente, podendo-se citar como exemplo os estudos de Sandström et al. ${ }^{22} \mathrm{e}$ de Peres et al. ${ }^{23}$.

Sandström et al. ${ }^{22}$ avaliaram os efeitos do ferro sobre o zinco em adultos através de uma 
solução teste (água, ácido ascórbico, sulfato ferroso e sulfato de zinco) e de uma refeição teste (arroz com molho de carne; ácido ascórbico, sulfato de zinco e sulfato ferroso) nas razões molares de $1: 1 ; 2,5: 1$ e 25:1. Na solução teste, até 2,5:1 não houve inibição. No entanto, quando a razão passou para 25:1, houve redução significativa na absorção do zinco, efeito este que foi diminuído quando a solução apresentava um ligante (histidina). Já na refeição teste, não houve redução significativa em nenhuma das razões molares. Assim sendo, parece que a ação inibitória pode ser diminuída na presença de ligantes de alimentos, que acabam por modificar a biodisponibilidade dos minerais.

Peres et al. ${ }^{23}$ estudaram a influência da relação ferro e zinco e da deficiência de ferro na absorção do zinco em ratos. Acima da razão molar de 2:1 houve inibição da absorção do zinco em grupos de ratos normais e com deficiência de ferro. Os dados apresentados pelos autores revelam que a inibição da absorção do zinco pelo ferro depende não só de suas quantidades, mas também do estado nutricional do indivíduo, ressaltando a importância de não se descuidar do zinco quando se inicia um programa de suplementação de ferro.

Em contrapartida, Rodriguez-M atas et al. ${ }^{24}$ demonstraram que numa situação de deficiência de ferro em ratos, a absorção de zinco permanece inalterada, provocando maiores alterações no metabolismo do cobre do que no zinco.

Todavia, os efeitos da suplementação de ferro sobre a biodisponibilidade de zinco de uma dieta regional em ratos também foram demonstrados por Pedrosa \& Cozzolino ${ }^{19}$. Neste estudo, o aumento da concentração de ferro na dieta (4 vezes o teor encontrado) interferiu negativamente no aproveitamento do zinco.

0 impacto da suplementação com ferro em um grupo de gestantes foi estudado por 0 'Brien et al. ${ }^{21}$, que observaram que a suplementação de ferro diminui a absorção de zinco em mais de $50 \%$, o que pode ter afetado adversamente 0 estado de zinco conforme evidenciado pela menor concentração plasmática deste elemento. Apesar da adição de $15 \mathrm{mg}$ de zinco no suplemento não influenciar significativamente a absorção de zinco, esta forneceu $3 \mathrm{mg}$ a mais de zinco absorvido por dia, quantidade suficiente para atender à demanda gestacional. Contudo, os autores concluem que, a inclusão de zinco nos suplementos pré-natais pode minimizar a interação entre ferro e zinco em populações com deficiência destes nutrientes.

Enquanto a suplementação de ferro parece exercer efetivamente uma inibição na absorção do zinco, estudos com alimentos fortificados não têm chegado aos mesmos resultados.

Haschke et al. ${ }^{25}$ verificaram a influência da fortificação de fórmulas infantis com ferro em duas concentrações $(10,2 \mathrm{mg} / \mathrm{L}$ e $2,5 \mathrm{mg} / \mathrm{L})$ sobre a absorção de elementos traços, mais especificamente o zinco, e não reportaram diferença na absorção deste mineral em crianças de 43 a 420 dias de idade. Da mesma forma, Fairweather-Tait et al. ${ }^{26}$ estudaram os efeitos de um alimento infantil fortificado com ferro e não observaram diferença na absorção aparente do zinco em crianças de 9 meses. Num estudo em adultos onde foram utilizados três diferentes alimentos fortificados (cereal infantil com $500 \mathrm{mg}$ de ferro por Kg; pão de trigo com 65mg de ferro por kg e uma fórmula infantil com $12 \mathrm{mg}$ de ferro por litro), Davidsson et al. ${ }^{27}$ também não reportaram diferença na absorção do zinco em relação a absorção quando da ingestão de alimentos não fortificados.

\section{O NSIDERAÇÃ O FINAL}

Apesar dos estudos apresentarem resultados controversos, o que pode ser explicado pelo emprego de diferentes metodologias, o uso de suplementos de minerais ou de alimentos fortificados deve levar em consideração possíveis interações entre minerais. É necessária a realização de novos estudos que venham a elucidar a questão das interações entre minerais. 


\section{REFERÊ N CIAS}

1. Williams SR. Fundamentos de nutrição e dietoterapia. Porto Alegre: Artmed Editora; 1997. $668 p$.

2. Cozzolino SM. Biodisponibilidade de minerais. $R$ Nutr PUCCAM P 1997; 10(2):87-98.

3. Bremner I, Beattie JH. Copper and zinc metabolism in health and disease: speciation and interactions. Proc Nutr Soc 1995; 54:489-99.

4. Couzi F, Keen C, Gershwin ME, Mareshi JP. Nutritional implications of the interactions between minerals. Progr Food Nutr Scie 1993; 17:65-87.

5. Dawson-Hughes B, Seligson FH, Hughes VA. Effects of calcium carbonate and hydroxyapatite on zinc and iron retention in postmenopausal women. Am J Clin Nutr 1986; 44:83-8.

6. Wood RJ, Zheng JJ. High dietary calcium intakes reduce zinc absorption and balance in humans. Am J Clin Nutr 1997; 65:1803-9.

7. Cook JD, Dassenko SA, Whittaker P. Calcium supplementation: effect on iron absorption. Am J Clin Nutr 1991; 53:106-11.

8. Gleerup A, Rossander-Hulthén L, Gramatkovski E, Hallber L. Iron absorption from the whole-diet: comparison of the effect of two different distributions of daily calcium intake. Am J Clin Nutr 1995; 61:97-104.

9. Hallberg $L$, Brune $M$, Erlandsson $M$, Sandberg $A$, Rossander-Hulthén L. Calcium: effect of different amounts on nonheme and heme-iron absorption in humans. Am J Clin Nutr 1991; 53:112-9.

10. Al-M asri MR. Absorption and endogenous excretion of phosphorus in growin broiler chiks, as influenced by calcium and phosphorus ratios in fed. Br J Nutr 1995; 74(3):407-15

11. Spencer $H$, Kramer L, Osis D. Effect of calcium on phosphorus metabolism in man. Am J Clin Nutr 1984; 40(2):219-25.

12. Walter A, Rimbach G, Most E, Pallauf J. Effects of calcium supplements to a maize-soya diet on the bioavailability of minerals and trace elements and the accumulation of heavy metals in growing rats. J Vet Med A Physiol Pathol Clin Med 2000; 47(6):367-77.

13. Reddy MB, Cook JD. Effect of calcium intake on nonheme-iron absorption from a complete diet. Am J Clin Nutr 1997; 65:1820-5.

14. Fairweather-Tait SJ. Iron-zinc and calcium-iron interactions in relation to $\mathrm{Zn}$ and Fe absorption. Proc Nutr Soc 1995; 54:465-73.

15. Yan L, Prentice A, Dibba B, Jarjou LMA, Stirling $D M$. The effect of long-term calcium supplementation on indices of iron, zinc and magnesium status in lactating Gambian women. Br J Nutr 1996; 76:821-31.

16. Fordyce EJ, Forbes RM, Robbins KR, Erdman JW. Phytate: calcium/zinc molar ratio: are they predective of zinc bioavailability? J Food Sci 1987; 52:440-4.

17. Lönnerdal B. Dietary factors influencing zinc absorption. J Nutr 2000; 130(Suppl):1378S-83S.

18. Lönnerdal B, Cederblad A, Davidsson L, Sandström $B$. The effect of individual components of soy formula and cow's milk formula on zinc bioavailability. Am J Clin Nutr 1984; 40: 1064-70.

19. Pedrosa LFC, Cozzolino SMF. Efeito da suplementação com ferro na biodisponibilidade de zinco em uma dieta regional do nordeste do Brasil. Rev Saúde Pública 1993; 27(4):266-70.

20. Whittaker P. Iron and zinc interactions in humans. Am J Clin Nutr 1998; 68(Suppl 2):442S-6S.

21. O'Brien KO, Zavaleta N, Caulfield LE, Wen J, Abrams SA. Prenatal iron supplements impair zinc absorption in pregnant peruvian women. J Nutr 2000; 130(9):2251-5.

22. Sandström B, Davidsson L, Cederblad A, Lönnerdal B. Oral iron, dietary ligands and zinc absorption. J Nutr 1985; 115:411-4.

23. Peres JM, Bureau F, Neuville D, Arhan P, Bougle D. Inibition of zinc absorption by iron depends on their ratio. J Trace Elem Med Biol 2001; 15(4): 237-41. 
24. Rodriguez-Matas M C, Lisbona F, Gomez-Ayala AE, Lopez-Aliaga I, Campos MS. Influence of nutritional iron deficiency development on some aspects of iron, copper and zinc metabolism. Lab Anim 1998; 32(3):298-306.

25. Haschke F, Ziegler EE, Edwards BB, Fomon SJ . Effect of iron fortification of infant fórmula on trace mineral absorption. J Pediatric Gastroenterol Nutr 1986; 5:768-73.
26. Fairweather-Tait SJ, Wharf SG, Fox TE. Zinc absorption in infants fed iron-fortified weaning food. Am J Clin Nutr 1995; 62:785-9.

27. Davidsson L, Almgren A, Sandströnm B, Hurrel RF. Zinc absorption in adult humans: the effect of iron fortification. Br J Nutr 1995 74(3):417-25.

Recebido para publicação em 11 de novembro de 2002 e aceito em 23 de abril de 2003. 
